\title{
Integro-differential inequality and stability of BAM FCNNs with time delays in the leakage terms and distributed delays
}

\author{
Xinhua Zhang and Kelin Li
}

\author{
* Correspondence: Ikl@suse.edu.cn \\ School of Science, Sichuan \\ University of Science \& \\ Engineering, Sichuan 643000, PR \\ China
}

\begin{abstract}
In this paper, a class of impulsive bidirectional associative memory (BAM) fuzzy cellular neural networks (FCNNs) with time delays in the leakage terms and distributed delays is formulated and investigated. By establishing an integro-differential inequality with impulsive initial conditions and employing M-matrix theory, some sufficient conditions ensuring the existence, uniqueness and global exponential stability of equilibrium point for impulsive BAM FCNNs with time delays in the leakage terms and distributed delays are obtained. In particular, the estimate of the exponential convergence rate is also provided, which depends on the delay kernel functions and system parameters. It is believed that these results are significant and useful for the design and applications of BAM FCNNs. An example is given to show the effectiveness of the results obtained here.

Keywords: bidirectional associative memory, fuzzy cellular neural networks, impulses, distributed delays, global exponential stability
\end{abstract}

\section{Introduction}

The bidirectional associative memory (BAM) neural network models were first introduced by Kosko [1]. It is a special class of recurrent neural networks that can store bipolar vector pairs. The BAM neural network is composed of neurons arranged in two layers, the $\mathrm{X}$-layer and Y-layer. The neurons in one layer are fully interconnected to the neurons in the other layer. Through iterations of forward and backward information flows between the two layer, it performs a two-way associative search for stored bipolar vector pairs and generalize the single-layer autoassociative Hebbian correlation to a two-layer patternmatched heteroassociative circuits. Therefore, this class of networks possesses good application prospects in some fields such as pattern recognition, signal and image process, and artificial intelligence [2]. In such applications, the stability of networks plays an important role; it is of significance and necessary to investigate the stability. It is well known, in both biological and artificial neural networks, the delays arise because of the processing of information. Time delays may lead to oscillation, divergence or instability which may be harmful to a system. Therefore, study of neural dynamics with consideration of the delayed problem becomes extremely important to manufacture high-quality neural networks. In recent years, there have been many analytical results for BAM neural networks with various axonal signal transmission delays, for example, see [3-11] and references therein. In addition, except various axonal signal transmission delays, time delay in the

(C) 2011 Zhang and Li; licensee Springer. This is an Open Access article distributed under the terms of the Creative Commons Attribution License (http://creativecommons.org/licenses/by/2.0), which permits unrestricted use, distribution, and reproduction in any medium, provided the original work is properly cited. 
leakage term also has great impact on the dynamics of neural networks. As pointed out by Gopalsamy [12,13], time delay in the stabilizing negative feedback term has a tendency to destabilize a system. Recently, some authors have paid attention to stability analysis of neural networks with time delays in the leakage (or "forgetting") terms [12-18].

Since FCNNs were introduced by Yang et. al $[19,20]$, many researchers have done extensive works on this subject due to their extensive applications in classification of image processing and pattern recognition. Specially, in the past few years, the stability analysis on FCNNs with various delays and fuzzy BAM neural networks with transmission delays has been the highlight in the neural network field, for example, see [21-27] and references therein. On the other hand, in respect of the complexity, besides delay effect, impulsive effect likewise exists in a wide variety of evolutionary processes in which states are changed abruptly at certain moments of time, involving such fields as medicine and biology, economics, mechanics, electronics and telecommunications. Many interesting results on impulsive effect have been gained, e.g., Refs. [28-37]. As artificial electronic systems, neural networks such as CNNs, bidirectional neural networks and recurrent neural networks often are subject to impulsive perturbations, which can affect dynamical behaviors of the systems just as time delays. Therefore, it is necessary to consider both impulsive effect and delay effect on the stability of neural networks. To the best of our knowledge, few authors have considered impulsive BAM FCNNs with time delays in the leakage terms and distributed delays.

Motivated by the above discussions, the objective of this paper is to formulate and study impulsive BAM FCNNs with time delays in the leakage terms and distributed delays. Under quite general conditions, some sufficient conditions ensuring the existence, uniqueness and global exponential stability of equilibrium point are obtained by the topological degree theory, properties of $M$-matrix, the integro-differential inequality with impulsive initial conditions and analysis technique.

The paper is organized as follows. In Section 2, the new neural network model is formulated, and the necessary knowledge is provided. The existence and uniqueness of equilibrium point are presented in Section 3. In Section 4, we give some sufficient conditions of exponential stability of the impulsive BAM FCNNs with time delays in the leakage terms and distributed delays. An example is given to show the effectiveness of the results obtained here in Section 5. Finally, in Section 6, we give the conclusion.

\section{Model description and preliminaries}

In this section, we will consider the model of impulsive BAM FCNNs with time delays in the leakage terms and distributed delays, it is described by the following functional differential equation:

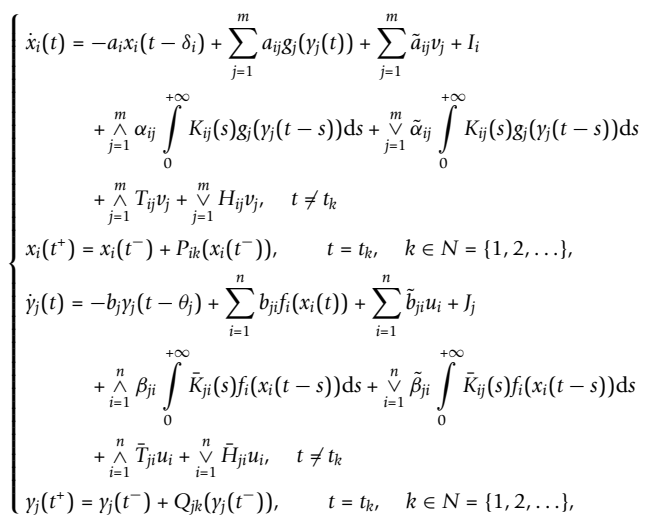


for $i=1,2, \ldots, n, j=1,2, \ldots, m, t>0$, where $x_{i}(t)$ and $y_{j}(t)$ are the states of the $i$ th neuron and the jth neuron at time $t$, respectively; $\delta_{i} \geq 0$ and $\theta_{j} \geq 0$ denote the leakage delays, respectively; $f_{i}$ and $g_{j}$ denote the signal functions of the $i$ th neuron and the $j$ th neuron at time $t$, respectively; $u_{i}, v_{j}$ and $I_{i}, J_{j}$ denote inputs and bias of the $i$ theuron and the $j$ th neuron, respectively; $a_{i}>0, b_{j}>0, a_{i j}, \tilde{a}_{i j}, \alpha_{i j}, \tilde{\alpha}_{i j}, b_{j i}, \tilde{b}_{j i}, \beta_{j i}, \tilde{\beta}_{j i}$ are constants, $a_{i}$ and $b_{j}$ represent the rate with which the $i$ th neuron and the $j$ th neuron will reset their potential to the resting state in isolation when disconnected from the networks and external inputs, respectively; $a_{i j}, b_{j i}$ and $\tilde{a}_{i j}, \tilde{b}_{j i}$ denote connection weights of feedback template and feedforward template, respectively; $\alpha_{i j}, \beta_{j i}$ and $\tilde{\alpha}_{i j}, \tilde{\beta}_{j i}$ denote connection weights of the distributed fuzzy feedback MIN template and the distributed fuzzy feedback MAX template, respectively; $T_{i j}, \bar{T}_{j i}$ and $H_{i j}, \tilde{H}_{j i}$ are elements of fuzzy feedforward MIN template and fuzzy feedforward MAX template, respectively; $\Lambda$ and $\vee$ denote the fuzzy AND and fuzzy OR operations, respectively; $K_{i j}(s)$ and $\bar{K}_{j i}(s)$ correspond to the delay kernel functions, respectively. $t_{k}$ is called impulsive moment and satisfies $0<t_{1}<t_{2}<\ldots, \lim _{k \rightarrow+\infty} t_{k}=+\infty ; x_{i}\left(t_{k}^{-}\right)$and $x_{i}\left(t_{k}^{+}\right)$denote the left-hand and righthand limits at $t_{k}$, respectively; $P_{i k}$ and $Q_{j k}$ show impulsive perturbations of the $i$ th neuron and $j$ th neuron at time $t_{k}$, respectively.

We always assume $x_{i}\left(t_{k}^{+}\right)=x_{i}\left(t_{k}\right)$ and $\gamma_{j}\left(t_{k}^{+}\right)=\gamma_{j}\left(t_{k}\right), k \in N$. The initial conditions are given by

$$
\left\{\begin{array}{l}
x_{i}(t)=\phi_{i}(t),-\infty \leq t \leq 0 \\
y_{j}(t)=\varphi_{j}(t),-\infty \leq t \leq 0
\end{array}\right.
$$

where $\varphi_{i}(t), \phi_{j}(t)(i=1,2, \ldots, n ; j=1,2, \ldots, m)$ are bounded and continuous on $(-\infty, 0]$, respectively.

If the impulsive operators $P_{i k}\left(x_{i}\right)=0, Q_{j k}\left(y_{j}\right)=0, i=1,2, \ldots, n, j=1,2, \ldots, m, k \in N$, then system (1) may reduce to the following model:

$$
\begin{aligned}
& \left\{\begin{aligned}
\dot{x}_{i}(t)= & -a_{i} x_{i}\left(t-\delta_{i}\right)+\sum_{j=1}^{m} a_{i j} g_{j}\left(\gamma_{j}(t)\right)+\sum_{j=1}^{m} \tilde{a}_{i j} v_{j}+I_{i} \\
& +\wedge_{j=1}^{m} \alpha_{i j} \int_{0}^{+\infty} K_{i j}(s) g_{j}\left(y_{j}(t-s)\right) \mathrm{d} s+{\underset{j=1}{m}}_{\tilde{\alpha}_{i j}}^{+\infty} \int_{0}^{+\infty} K_{i j}(s) g_{j}\left(y_{j}(t-s)\right) \mathrm{d} s
\end{aligned}\right. \\
& +\bigwedge_{j=1}^{m} T_{i j} v_{j}+\underbrace{m}_{j=1} H_{i j} v_{j}, \\
& \dot{y}_{j}(t)=-b_{j} y_{j}\left(t-\theta_{j}\right)+\sum_{i=1}^{n} b_{j i} f_{i}\left(x_{i}(t)\right)+\sum_{i=1}^{n} \tilde{b}_{j i} u_{i}+J_{j} \\
& +\wedge_{i=1}^{n} \beta_{j i} \int_{0}^{+\infty} \bar{K}_{j i}(s) f_{i}\left(x_{i}(t-s)\right) \mathrm{d} s+\bigvee_{i=1}^{n} \tilde{\beta}_{j i} \int_{0}^{+\infty} \bar{K}_{i j}(s) f_{i}\left(x_{i}(t-s)\right) \mathrm{d} s \\
& +\bigwedge_{i=1}^{n} \bar{T}_{j i} u_{i}+\bigvee_{i=1}^{n} \bar{H}_{j i} u_{i} .
\end{aligned}
$$

System (2) is called the continuous system of model (1).

Throughout this paper, we make the following assumptions: 
(H1) For neuron activation functions $f_{i}$ and $g_{j}(i=1,2, \ldots, n ; j=1,2, \ldots, m)$, there exist two positive diagonal matrices $F=\operatorname{diag}\left(F_{1}, F_{2}, \ldots, F_{n}\right)$ and $G=\operatorname{diag}\left(G_{1}, G_{2}, \ldots\right.$, $\left.G_{m}\right)$ such that

$$
F_{i}=\sup _{x \neq y}\left|\frac{f_{i}(x)-f_{i}(y)}{x-y}\right|, \quad G_{j}=\sup _{x \neq y}\left|\frac{g_{j}(x)-g_{j}(y)}{x-y}\right|
$$

for all $x, y \in R(x \neq y)$.

$(\mathrm{H} 2)$ The delay kernels $K_{i j}:[0,+\infty) \rightarrow R$ and $\bar{K}_{j i}:[0,+\infty) \rightarrow R$ are real-valued piecewise continuous, and there exists $\delta>0$ such that

$$
k_{i j}(\lambda)=\int_{0}^{+\infty} \mathrm{e}^{\lambda s}\left|K_{i j}(s)\right| \mathrm{d} s, \quad \bar{k}_{j i}(\lambda)=\int_{0}^{+\infty} \mathrm{e}^{\lambda s}\left|\bar{K}_{j i}(s)\right| \mathrm{d} s
$$

Are continuous for $\lambda \in[0, \delta), i=1,2, \ldots, n, j=1,2, \ldots, m$.

(H3) Let $\bar{P}_{k}(x)=x+P_{k}(x)$ and $\bar{Q}_{k}(\gamma)=y+Q_{k}(\gamma)$ be Lipschitz continuous in $R^{n}$ and $R^{m}$, respectively, that is, there exist nonnegative diagnose matrices $\Gamma_{k}=\operatorname{diag}\left(\gamma_{1 k}, \gamma_{2 k}, \ldots\right.$, $\left.\gamma_{n k}\right)$ and $\bar{\Gamma}_{k}=\operatorname{diag}\left(\bar{\gamma}_{1 k}, \bar{\gamma}_{2 k}, \ldots, \bar{\gamma}_{m k}\right)$ such that

$$
\begin{aligned}
& \left|\bar{P}_{k}(x)-\bar{P}_{k}(y)\right| \leq \Gamma_{k}|x-y|, \quad \text { for all } x, y \in R^{n}, \quad k \in N, \\
& \left|\bar{Q}_{k}(u)-\bar{Q}_{k}(v)\right| \leq \bar{\Gamma}_{k}|u-v|, \quad \text { for all } u, v \in R^{m}, \quad k \in N,
\end{aligned}
$$

where

$$
\begin{aligned}
& \bar{P}_{k}(x)=\left(\bar{P}_{1 k}\left(x_{1}\right), \bar{P}_{2 k}\left(x_{2}\right), \ldots, \bar{P}_{n k}\left(x_{n}\right)\right)^{T}, \\
& \bar{Q}_{k}(x)=\left(\bar{Q}_{1 k}\left(y_{1}\right), \bar{Q}_{2 k}\left(y_{2}\right), \ldots, \bar{Q}_{m k}\left(y_{m}\right)\right)^{T}, \\
& P_{k}(x)=\left(P_{1 k}\left(x_{1}\right), P_{2 k}\left(x_{2}\right), \ldots, P_{n k}\left(x_{n}\right)\right)^{T}, \\
& Q_{k}(y)=\left(Q_{1 k}\left(y_{1}\right), Q_{2 k}\left(y_{2}\right), \ldots, Q_{m k}\left(y_{m}\right)\right)^{T} .
\end{aligned}
$$

To begin with, we introduce some notation and recall some basic definitions.

$P C\left[, R^{l}\right]=\left\{z(t): J \rightarrow R^{l} \mid z(t)\right.$ is continuous at $t \neq t_{k}, z\left(t_{k}^{+}\right)=z\left(t_{k}\right)$, and $z\left(t_{k}^{-}\right)$exists for $t$, $\left.t_{k} \in J, k \in N\right\}$, where $J \subset R$ is an interval, $l \in N$.

$P C=\left\{\psi:(-\infty, 0] \rightarrow R^{l} \mid \psi(s)\right.$ is bounded, and $\psi\left(s^{+}\right)=\psi(s)$ for $s \in(-\infty, 0), \psi\left(s^{-}\right)$exists for $s \in(-\infty, 0], \varphi\left(s^{-}\right)=\varphi(s)$ for all but at most a finite number of points $\left.s \in(-\infty, 0]\right\}$.

For an $m \times n$ matrix $A,|A|$ denotes the absolute value matrix given by $|A|=\left(\left|a_{i j}\right|\right)_{m}$ ${ }_{\times n}$. For $A=\left(a_{i j}\right)_{m \times n}, B=\left(b_{i j}\right)_{m \times n} \in R^{m \times n}, A \geq B(A>B)$ means that each pair of corresponding elements of $A$ and $B$ such that the inequality $a_{i j} \geq b_{i j}\left(a_{i j}>b_{i j}\right)$.

Definition 1 A function $(x, y)^{T}:(-\infty,+\infty) \rightarrow R^{n+m}$ is said to be the special solution of system (1) with initial conditions

$$
x(s)=\phi(s), \quad \gamma(s)=\varphi(s) \quad s \in(-\infty, 0],
$$

if the following two conditions are satisfied

(i) $(x, y)^{T}$ is piecewise continuous with first kind discontinuity at the points $t_{k}, k \in K$. Moreover, $(x, y)^{T}$ is right continuous at each discontinuity point.

(ii) $(x, y)^{T}$ satisfies model (1) for $t \geq 0$, and $x(s)=\varphi(s), y(s)=\phi(s)$ for $s \in(-\infty, 0]$.

Especially, a point $\left(x^{*}, y^{*}\right)^{T} \in R^{n+m}$ is called an equilibrium point of model (1), if (x $(t), y(t))^{T}=\left(x^{*}, y^{*}\right)^{T}$ is a solution of $(1)$.

Throughout this paper, we always assume that the impulsive jumps $P_{k}$ and $Q_{k}$ satisfy (referring to [28-37]) 


$$
P_{k}\left(x^{*}\right)=0 \quad \text { and } \quad Q_{k}\left(y^{*}\right)=0, \quad k \in N,
$$

i.e.,

$$
\bar{P}_{k}\left(x^{*}\right)=x^{*} \quad \text { and } \quad \bar{Q}\left(\gamma^{*}\right)=y^{*}, \quad k \in N,
$$

where $\left(x^{*}, y^{*}\right)^{T}$ is the equilibrium point of continuous systems (2). That is, if $\left(x^{*}, y^{*}\right)^{T}$ is an equilibrium point of continuous system (2), then $\left(x^{*}, y^{*}\right)^{T}$ is also the equilibrium of impulsive system (1).

Definition 2 The equilibrium point $\left(x^{*}, y^{*}\right)^{T}$ of model (1) is said to be globally exponentially stable, if there exist constants $\lambda>0$ and $M \geq 1$ such that

$$
\left\|x(t)-x^{*}\right\|+\left\|y(t)-y^{*}\right\| \leq M\left(\left\|\phi-x^{*}\right\|+\left\|\varphi-y^{*}\right\|\right) e^{-\lambda t}
$$

for all $t \geq 0$, where $(x(t), y(t))^{T}$ is any solution of system (1) with initial value $(\varphi(s)$, $\phi(s))^{T}$ and

$$
\begin{aligned}
& \left\|x(t)-x^{*}||=\sum_{i=1}^{n}\left|x_{i}(t)-x_{i}^{*}\right|, \quad\right\| y(t)-y^{*} \|=\sum_{j=1}^{m}\left|y_{j}(t)-y_{j}^{*}\right|, \\
& \left\|\phi-x^{*}\right\|=\sup _{-\infty<s \leq 0} \sum_{i=1}^{n}\left|\phi_{i}(s)-x_{i}^{*}\right|, \quad\left\|\varphi-\gamma^{*}\right\|=\sup _{-\infty<s \leq 0} \sum_{j=1}^{m}\left|\varphi_{j}(s)-y_{j}^{*}\right| .
\end{aligned}
$$

Definition 3 A real matrix $D=\left(d_{i j}\right)_{n \times n}$ is said to be a nonsingular M-matrix if $d_{i j} \leq$ $0, i, j=1,2, \ldots, n, i \neq j$, and all successive principal minors of $D$ are positive.

Lemma 1 [38]Let $D=\left(d_{i j}\right)_{n \times n}$ with $d_{i j} \leq 0(i \neq j)$, then the following statements are true:

(i) $D$ is a nonsingular $M$-matrix if and only if $D$ is inverse-positive, that is, $D^{-1}$ exists and $D^{-1}$ is a nonnegative matrix.

(ii) $D$ is a nonsingular M-matrix if and only if there exists a positive vector $\xi=\left(\xi_{1}, \xi_{2}\right.$, ..., $\left.\xi_{n}\right)^{T}$ such that $D \xi>0$.

Lemma 2 [20]For any positive integer $n$, let $h_{j}: R \rightarrow R$ be a function $(j=1,2, \ldots, n)$, then we have

$$
\begin{aligned}
& \left|\bigwedge_{j=1}^{n} \alpha_{j} h_{j}\left(u_{j}\right)-\bigwedge_{j=1}^{n} \alpha_{j} h_{j}\left(v_{j}\right)\right| \leq \sum_{j=1}^{n}\left|\alpha_{j}\right| \cdot\left|h_{j}\left(u_{j}\right)-h_{j}\left(v_{j}\right)\right|, \\
& \left|\vee_{j=1}^{n} \alpha_{j} h_{j}\left(u_{j}\right)-\bigvee_{j=1}^{n} \alpha_{j} h_{j}\left(v_{j}\right)\right| \leq \sum_{j=1}^{n}\left|\alpha_{j}\right| \cdot\left|h_{j}\left(u_{j}\right)-h_{j}\left(v_{j}\right)\right|
\end{aligned}
$$

for all $\alpha=\left(\alpha_{1}, \alpha_{2}, \ldots, \alpha_{n}\right)^{T}, u=\left(u_{1}, u_{2}, \ldots, u_{n}\right)^{T}, v=\left(v_{1}, v_{2}, \ldots, v_{n}\right)^{T} \in R^{n}$

\section{Existence and uniqueness of equilibrium point}

In this section, we will proof the existence and uniqueness of equilibrium point of model (1). For the sake of simplification, let

$$
\left\{\begin{array}{l}
\tilde{I}_{i}=\sum_{j=1}^{m} \tilde{a}_{i j} v_{j}+I_{i}+\bigwedge_{j=1}^{m} T_{i j} v_{j}+\bigvee_{j=1}^{m} H_{i j} v_{j}, i=1,2, \ldots, n, \\
\tilde{J}_{j}=\sum_{i=1}^{n} \tilde{b}_{j i} u_{i}+J_{j}+\bigwedge_{i=1}^{n} \bar{T}_{j i} u_{i}+\bigvee_{i=1}^{n} \bar{H}_{j i} u_{i}, j=1,2, \ldots, m,
\end{array}\right.
$$


then model (2) is reduced to

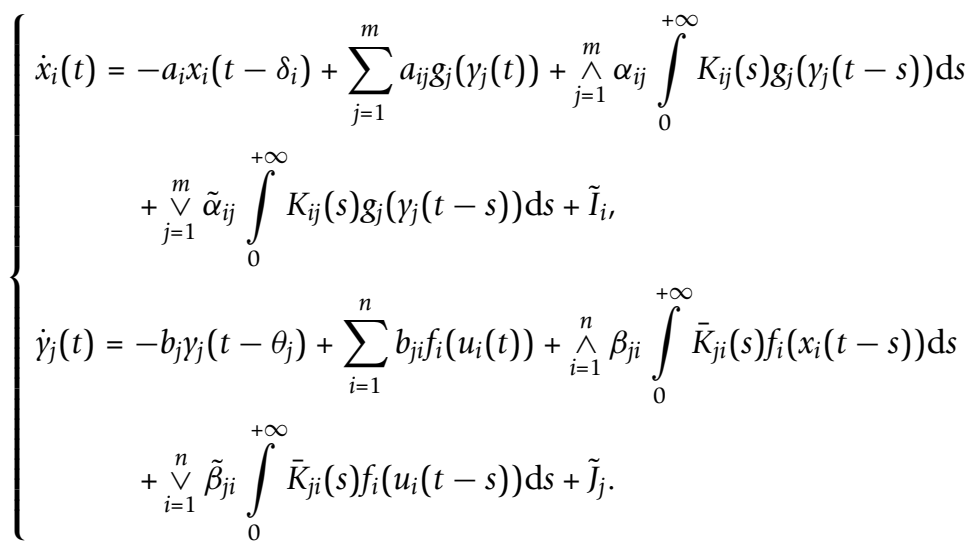

It is evident that the dynamical characteristics of model (2) are as same as of model (4).

Theorem 1 Under assumptions (H1) and (H2), system (1) has one unique equilibrium point, if the following condition holds,

(C1) there exist vectors $\xi=\left(\xi_{1}, \xi_{2}, \ldots, \xi_{n}\right)^{T}>0, \eta=\left(\eta_{1}, \eta_{2}, \ldots, \eta_{m}\right)^{T}>0$ and positive number $\lambda>0$ such that

$$
\left\{\begin{array}{l}
\left(\lambda-a_{i} e^{\lambda \delta_{i}}\right) \xi_{i}+\sum_{j=1}^{m}\left[\left|a_{i j}\right|+\left(\left|\alpha_{i j}\right|+\left|\tilde{\alpha}_{i j}\right|\right) k_{i j}(\lambda)\right] G_{j} \eta_{j}<0, i=1,2, \ldots, n, \\
\left(\lambda-b_{j} e^{\lambda \theta_{j}}\right) \eta_{j}+\sum_{i=1}^{n}\left[\left|b_{j i}\right|+\left(\left|\beta_{j i}\right|+\left|\tilde{\beta}_{j i}\right|\right) \bar{k}_{j i}(\lambda)\right] F_{i} \xi_{i}<0 . j=1,2, \ldots, m .
\end{array}\right.
$$

Proof. Let $h\left(x_{1}, \ldots, x_{n}, y_{1}, \ldots, y_{m}\right)=\left(h_{1}, \ldots, h_{n}, \bar{h}_{1}, \ldots, \bar{h}_{m}\right)^{T}$, where

$$
\left\{\begin{array}{l}
h_{i}=a_{i} x_{i}-\sum_{j=1}^{m} a_{i j} g_{j}\left(y_{j}\right)-\bigwedge_{j=1}^{m} \alpha_{i j} k_{i j}(0) g_{j}\left(y_{j}\right)-{\underset{j=1}{\vee}}_{j}^{m} \tilde{\alpha}_{i j} k_{i j}(0) g_{j}\left(y_{j}\right)-\tilde{I}_{i} \\
\bar{h}_{j}=b_{j} y_{j}-\sum_{i=1}^{n} b_{j i} f_{i}\left(x_{i}\right)-\bigwedge_{i=1}^{n} \beta_{j i} \bar{k}_{j i}(0) f_{i}\left(x_{i}\right)-\underbrace{n}_{i=1} \tilde{\beta}_{j i} \bar{k}_{j i}(0) f_{i}\left(x_{i}\right)-\tilde{J}_{j}
\end{array}\right.
$$

for $i=1,2, \ldots, n ; j=1,2, \ldots, m$. Obviously, from assumption (H2), the equilibrium points of model (4) are the solutions of system of equations:

$$
\left\{\begin{array}{l}
h_{i}=0, i=1,2, \ldots, n, \\
\bar{h}_{j}=0, j=1,2, \ldots, m .
\end{array}\right.
$$

Define the following homotopic mapping:

$H\left(x_{1}, \ldots, x_{n}, y_{1}, \ldots, y_{m}\right)=\theta h\left(x_{1}, \ldots, x_{n}, y_{1}, \ldots, y_{m}\right)+(1-\theta)\left(x_{1}, \ldots, x_{n}, y_{1}, \ldots, y_{m}\right)^{T}$, where $\theta$ $\in[0,1]$. Let $H_{k}(k=1,2, \ldots, n+m)$ denote the $k$ th component of $H\left(x_{1}, \ldots, x_{n}, y_{1}, \ldots, y_{m}\right)$, then from assumption (H1) and Lemma 2, we have

$$
\left\{\begin{aligned}
\left|H_{i}\right| \geq & {\left[1+\theta\left(a_{i}-1\right)\right]\left|x_{i}\right|-\theta \sum_{j=1}^{m}\left[\left|a_{i j}\right|+\left(\left|\alpha_{i j}\right|+\left|\tilde{\alpha}_{i j}\right|\right) k_{i j}(0)\right] G_{j}\left|y_{j}\right| } \\
& -\theta \sum_{j=1}^{m}\left[\left|a_{i j}\right|+\left(\left|\alpha_{i j}\right|+\left|\tilde{\alpha}_{i j}\right|\right) k_{i j}(0)\right]\left|\delta_{j}(0)\right|-\theta\left|\tilde{I}_{i}\right|, \\
\left|H_{n+j}\right| \geq & {\left[1+\theta\left(b_{j}-1\right)\right]\left|\gamma_{j}\right|-\theta \sum_{i=1}^{n}\left[\left|b_{j i}\right|+\left(\left|\beta_{j i}\right|+\left|\tilde{\beta}_{j i}\right|\right) \bar{k}_{j i}(0)\right] F_{i}\left|x_{i}\right| } \\
& -\theta \sum_{i=1}^{n}\left[\left|b_{j i}\right|+\left(\left|\beta_{j i}\right|+\left|\tilde{\beta}_{j i}\right|\right) \bar{k}_{j i}(0)\right]\left|f_{i}(0)\right|-\theta\left|\tilde{J}_{j}\right|
\end{aligned}\right.
$$


for $i=1,2, \ldots, n, j=1,2, \ldots, m$. Denote

$$
\begin{aligned}
\bar{H} & =\left(\left|H_{1}\right|,\left|H_{2}\right|, \ldots,\left|H_{n+m}\right|\right)^{T}, \quad z=\left(\left|x_{1}\right|, \ldots,\left|x_{n}\right|,\left|y_{1}\right|, \ldots,\left|y_{m}\right|\right)^{T}, \\
C & =\operatorname{diag}\left(a_{1}, \ldots, a_{n}, b_{1}, \ldots, b_{m}\right), \quad L=\operatorname{diag}\left(F_{1}, \ldots, F_{n}, G_{1}, \ldots, G_{m}\right), \\
P & =\left(\left|\tilde{I}_{1}\right|, \ldots,\left|\tilde{I}_{n},\right|,\left|\tilde{J}_{1}\right|, \ldots,\left|\tilde{J}_{m}\right|\right)^{T} \\
Q & =\left(\left|f_{1}(0)\right|, \ldots,\left|f_{n}(0)\right|,\left|g_{1}(0)\right|, \ldots,\left|g_{m}(0)\right|\right)^{T}, \\
A & =\left(\left|a_{i j}\right|+\left(\left|\alpha_{i j}\right|+\left|\tilde{\alpha}_{i j}\right|\right) k_{i j}(0)\right)_{n \times m^{\prime}} \quad B=\left(\left|b_{j i}\right|+\left(\left|\beta_{j i}\right|+\left|\tilde{\beta}_{j i}\right|\right) \bar{k}_{j i}(0)\right)_{m \times n^{\prime}}, \\
T & =\left(\begin{array}{cc}
0 & A \\
B & 0
\end{array}\right), \quad \omega=\left(\xi_{1}, \ldots, \xi_{n}, \eta_{1}, \ldots, \eta_{m}\right)^{T}>0 .
\end{aligned}
$$

Then, the matrix form of (6) is

$$
\bar{H} \geq[E+\theta(C-E)] z-\theta T L z-\theta(P+T Q)=(1-\theta) z+\theta[(C-T L) z-(P+T Q)]
$$

Since condition $(\mathrm{C} 1)$ holds, and $k_{i j}(\lambda), \bar{k}_{j i}(\lambda)$ are continuous on $[0, \delta)$, when $\lambda=0$ in (C1), we obtain

$$
\left\{\begin{array}{l}
-a_{i} \xi_{i}+\sum_{j=1}^{m}\left[\left|a_{i j}\right|+\left(\left|\alpha_{i j}\right|+\left|\tilde{\alpha}_{i j}\right|\right) k_{i j}(0)\right] G_{j} \eta_{j}<0, \quad i=1,2, \ldots, n \\
-b_{j} \eta_{j}+\sum_{i=1}^{n}\left[\left|b_{j i}\right|+\left(\left|\beta_{j i}\right|+\left|\tilde{\beta}_{j i}\right|\right) \bar{k}_{j i}(0)\right] F_{i} \xi_{i}<0 . \quad j=1,2, \ldots, m .
\end{array}\right.
$$

or in matrix form,

$$
(-C+T L) \omega<0
$$

From Lemma 1 , we know that $C-T L$ is a nonsingular $M$-matrix, so $(C-T L)^{-1}$ is a nonnegative matrix. Let

$$
\Gamma=\left\{z=\left(x_{1}, \ldots, x_{n}, y_{1}, \ldots, y_{m}\right)^{T} \mid z \leq \omega+(C-T L)^{-1}(P+T Q)\right\}
$$

then $\Gamma$ is nonempty, and from (6), for any $z=\left(x_{1}, \ldots, x_{n}, y_{1}, \ldots, y_{m}\right)^{T} \in \partial \Gamma$, we have

$$
\begin{aligned}
\bar{H} & \geq(1-\theta) z+\theta(C-T L)\left[z-(C-T L)^{-1}(P+T Q)\right] \\
& =(1-\theta)\left[\omega+(C-T L)^{-1}(P+T Q)\right]+\theta(C-T L) \omega>0, \quad \theta \in[0,1] .
\end{aligned}
$$

Therefore, for any $\left(x_{1}, \ldots, x_{n}, y_{1}, \ldots, y_{m}\right)^{T} \in \partial \Gamma$ and $\theta \in[0,1]$, we have $H \neq 0$. From homotopy invariance theorem [39], we get

$$
\operatorname{deg}(h, \Gamma, 0)=\operatorname{deg}(H, \Gamma, 0)=1,
$$

by topological degree theory, we know that (5) has at least one solution in $\Gamma$. That is, model (4) has at least an equilibrium point.

Now, we show that the solution of the system of Equations (5) is unique. Assume that $\left(x_{1}^{*}, \ldots, x_{n}^{*}, \gamma_{1}^{*}, \ldots, y_{m}^{*}\right)^{T}$ and $\left(\hat{x}_{1}, \ldots, \hat{x}_{n}, \hat{y}_{1}, \ldots, \hat{y}_{m}\right)^{T}$ are two solutions of the system of Equations (5), then 


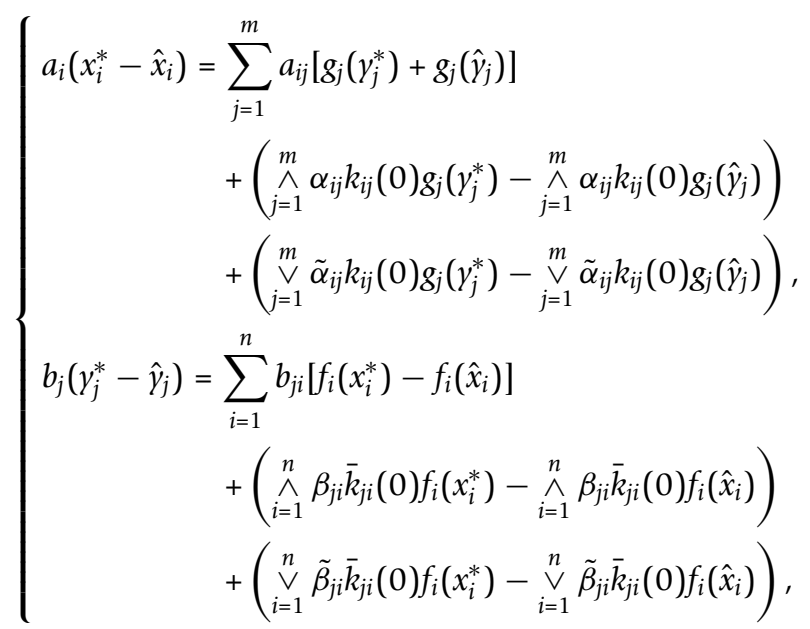

it follows that

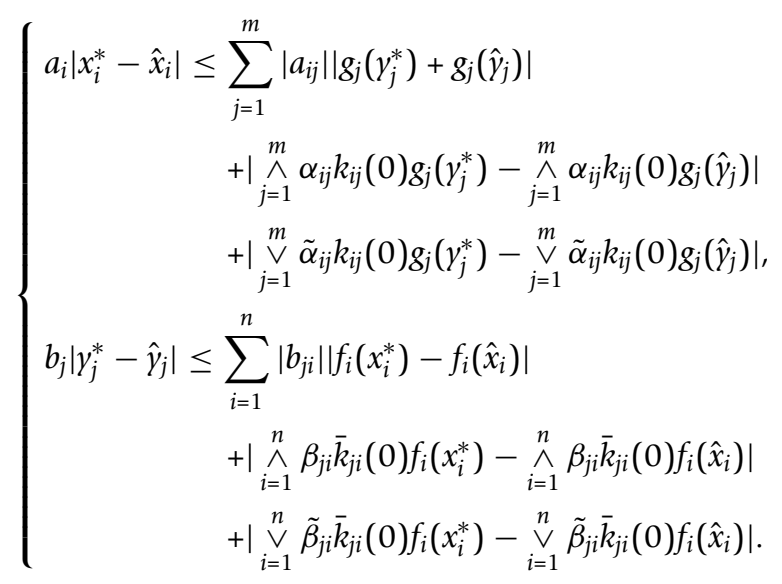

By using of Lemma 2 and hypothesis (H1), we have

$$
\left\{\begin{array}{l}
a_{i}\left|x_{i}^{*}-\hat{x}_{i}\right|-\sum_{j=1}^{m}\left[\left|a_{i j}\right|+\left(\left|\alpha_{i j}\right|+\left|\tilde{\alpha}_{i j}\right|\right) k_{i j}(0)\right] G_{j}\left|y_{j}^{*}-\hat{y}_{j}\right| \leq 0, \\
b_{j}\left|y_{j}^{*}-\hat{\gamma}_{j}\right|-\sum_{i=1}^{n}\left[\left|b_{j i}\right|+\left(\left|\beta_{j i}\right|+\left|\tilde{\beta}_{j i}\right|\right) \bar{k}_{j i}(0)\right] F_{i}\left|x_{i}^{*}-\hat{x}_{i}\right| \leq 0 .
\end{array}\right.
$$

Let $\left.Z=\operatorname{diag}_{(}\left|x_{1}^{*}-\hat{x}_{1}\right|, \ldots,\left|x_{n}^{*}-\hat{x}_{n}\right|,\left|y_{1}^{*}-\hat{y}_{1}\right|, \ldots,\left|y_{m}^{*}-\hat{y}_{m}\right|\right)$, then the matrix form of (8) is $(C-T L) Z \leq 0$. Since $C-T L$ is a nonsingular $M$-matrix, $(C-T L)^{-1} \geq 0$, thus $Z \leq$ 0 , accordingly, $Z=0$, i.e., $x_{i}^{*}=\hat{x}_{i}, y_{j}^{*}=\hat{y}_{j}(i=1,2, \ldots, n, j=1,2, \ldots, m)$. This shows that model (4) has one unique equilibrium point. According to (3), this implies that system (1) has one unique equilibrium point. The proof is completed.

Corollary 1 Under assumptions (H1) and (H2), system (1) has one unique equilibrium point if $C$ - TL is a nonsingular M-matrix.

Proof. Since that $C-T L$ is a nonsingular $M$-matrix, from Lemma 1 , there exists a vector $\omega=\left(\xi_{1}, \ldots \xi_{n}, \eta_{1}, \ldots, \eta_{m}\right)^{T}>0$ such that $(C T L) \omega>0$, or $(-C+T L) \omega<0$. It follows that 


$$
\left\{\begin{array}{l}
-a_{i} \xi_{i}+\sum_{j=1}^{m}\left[\left|a_{i j}\right|+\left(\left|\alpha_{i j}\right|+\left|\tilde{\alpha}_{i j}\right|\right) k_{i j}(0)\right] G_{j} \eta_{j}<0, \quad i=1,2, \ldots, n, \\
-b_{j} \eta_{j}+\sum_{i=1}^{n}\left[\left|b_{j i}\right|+\left(\left|\beta_{j i}\right|+\left|\tilde{\beta}_{j i}\right|\right) \bar{k}_{j i}(0)\right] F_{i} \xi_{i}<0, \quad j=1,2, \ldots, m .
\end{array}\right.
$$

From the continuity of $k_{i j}(\lambda)$ and $\bar{k}_{j i}(\lambda)$, it is easy to know that there exists $\lambda>0$ such that

$$
\begin{cases}\left(\lambda-a_{i} e^{\lambda \delta_{i}}\right) \xi_{i}+\sum_{j=1}^{m}\left[\left|a_{i j}\right|+\left(\left|\alpha_{i j}\right|+\left|\tilde{\alpha}_{i j}\right|\right) k_{i j}(\lambda)\right] G_{j} \eta_{j}<0, & i=1,2, \ldots, n, \\ \left(\lambda-b_{j} e^{\lambda \theta_{j}}\right) \eta_{j}+\sum_{i=1}^{n}\left[\left|b_{j i}\right|+\left(\left|\beta_{j i}\right|+\left|\tilde{\beta}_{j i}\right|\right) \bar{k}_{j i}(\lambda)\right] F_{i} \xi_{i}<0, & j=1,2, \ldots, m .\end{cases}
$$

That is, condition (C1) holds. This completes the proof.

\section{Exponential stability and exponential convergence rate}

In this section, we will discuss the global exponential stability of system (1) and give an estimation of exponential convergence rate.

Lemma 3 Let $a<b \leq+\infty$, and $u(t)=\left(u_{1}(t), \ldots, u_{n}(t)\right)^{T} \in P C\left[[a, b), R^{n}\right]$ and $v(t)=\left(v_{1}\right.$ $\left.(t), \ldots, v_{m}(t)\right)^{T} \in P C\left[[a, b), R^{m}\right]$ satisfy the following integro-differential inequalities with the initial conditions $u(s) \in P C\left[(-\infty, 0], R^{n}\right]$ and $v(s) \in P C\left[(-\infty, 0], R^{m}\right]$ :

$$
\left\{\begin{array}{l}
D^{+} u_{i}(t) \leq-r_{i} u_{i}\left(t-\delta_{i}\right)+\sum_{j=1}^{m} p_{i j} v_{j}(t)+\sum_{j=1}^{m} q_{i j} \int_{0}^{+\infty}\left|K_{i j}(s)\right| v_{j}(t-s) \mathrm{d} s \\
D^{+} v_{j}(t) \leq-\bar{r}_{j} v_{j}\left(t-\theta_{j}\right)+\sum_{i=1}^{n} \bar{p}_{j i} u_{i}(t)+\sum_{i=1}^{n} \bar{q}_{j i} \int_{0}^{+\infty}\left|\bar{K}_{j i}(s)\right| u_{i}(t-s) \mathrm{d} s
\end{array}\right.
$$

for $i=1,2, \ldots, n, j=1,2, \ldots$, , where $r_{i}>0, p_{i j}>0, q_{i j}>0, \bar{r}_{j}>0, \bar{p}_{j i}>0, \bar{q}_{j i}>0, i=$ $1,2, \ldots, n, j=1,2, \ldots, m$. If the initial conditions satisfy

$$
\begin{cases}u(s) \leq \kappa \xi \mathrm{e}^{-\lambda(s-a)}, & s \in(-\infty, a] \\ v(s) \leq \kappa \eta \mathrm{e}^{-\lambda(s-a)}, & s \in(-\infty, a]\end{cases}
$$

in which $\lambda>0, \xi=\left(\xi_{1}, \xi_{2}, \ldots, \xi_{n}\right)^{T}>0$ and $\eta=\left(\eta_{1}, \eta_{2}, \ldots, \eta_{m}\right)^{T}>0$ satisfy

$$
\left\{\begin{array}{l}
\left(\lambda-r_{i} e^{\lambda \delta_{i}}\right) \xi_{i}+\sum_{j=1}^{m}\left(p_{i j}+q_{i j} k_{i j}(\lambda)\right) \eta_{j}<0, i=1,2, \ldots, n, \\
\left(\lambda-\bar{r}_{j} e^{\lambda \theta_{j}}\right) \eta_{j}+\sum_{i=1}^{n}\left(\bar{p}_{j i}+\bar{q}_{j i} \bar{k}_{j i}(\lambda)\right) \xi_{i}<0, j=1,2, \ldots, m .
\end{array}\right.
$$

Then

$$
\begin{cases}u(t) \leq \kappa \xi \mathrm{e}^{-\lambda(t-a)}, & t \in[a, b), \\ v(t) \leq \kappa \eta \mathrm{e}^{-\lambda(t-a)}, & t \in[a, b) .\end{cases}
$$

Proof. For $i \in\{1,2, \ldots, n\}, j \in\{1,2, \ldots, m\}$ and arbitrary $\varepsilon>0$, set $z_{i}(t)=(\kappa+\varepsilon) \xi_{i} e^{-\lambda}$ ${ }^{(t-a)}, \bar{z}_{j}(t)=(\kappa+\varepsilon) \eta_{j} \mathrm{e}^{-\lambda(t-a)}$, we prove that 


$$
\left\{\begin{array}{lll}
u_{i}(t) \leq z_{i}(t)=(\kappa+\varepsilon) \xi_{i} \mathrm{e}^{-\lambda(t-a),} & t \in[a, b), & i=1,2, \ldots, n, \\
v_{j}(t) \leq \bar{z}_{j}(t)=(\kappa+\varepsilon) \eta_{j} \mathrm{e}^{-\lambda(t-a),} & t \in[a, b), & j=1,2, \ldots, m .
\end{array}\right.
$$

If this is not true, no loss of generality, suppose that there exist $i_{0}$ and $t^{*} \in[a, b)$ such that

$$
u_{i_{0}}\left(t^{*}\right)=z_{i_{0}}\left(t^{*}\right), D^{+} u_{i_{0}}\left(t^{*}\right) \geq \dot{z}_{i_{0}}\left(t^{*}\right), u_{i}(t) \leq z_{i}(t), v_{j}(t) \leq \bar{z}_{j}(t)
$$

for $t \in\left[a, t^{*}\right], i=1,2, \ldots, n, j=1,2, \ldots, m$.

However, from (9) and (12), we get

$$
\begin{aligned}
& D^{+} u_{i_{0}}\left(t^{*}\right) \\
\leq & -r_{i_{0}} u_{i_{0}}\left(t^{*}-\delta_{i_{0}}\right)+\sum_{j=1}^{m} p_{i_{0} j} v_{j}\left(t^{*}\right)+\sum_{j=1}^{m} q_{i_{0} j} \int_{0}^{+\infty}\left|K_{i_{0} j}(s)\right| v_{j}\left(t^{*}-s\right) \mathrm{d} s \\
\leq & -r_{i_{0}}(\kappa+\varepsilon) \xi_{i_{0}} \mathrm{e}^{-\lambda\left(t^{*}-\delta_{i_{0}}-a\right)}+\sum_{j=1}^{m} p_{i_{0} j} \eta_{j}(\kappa+\varepsilon) \eta_{j} \mathrm{e}^{-\lambda\left(t^{*}-a\right)} \\
& +\sum_{j=1}^{m} q_{i_{0} j}(\kappa+\varepsilon) \eta_{j} e^{-\lambda\left(t^{*}-a\right)} \int_{0}^{+\infty} \mathrm{e}^{\lambda s}\left|K_{i_{0} j}(s)\right| \mathrm{d} s \\
= & {\left[-r_{i_{0}} \xi_{i_{0}} \mathrm{e}^{\lambda \delta_{i_{0}}}+\sum_{j=1}^{m}\left(p_{i_{0} j}+q_{i_{0} j} k_{i_{0} j}(\lambda)\right) \eta_{j}\right](\kappa+\varepsilon) \mathrm{e}^{-\lambda\left(t^{*}-a\right) .} }
\end{aligned}
$$

Since (11) holds, it follows that $-r_{i_{0}} \xi_{i_{0}} \mathrm{e}^{\lambda \delta_{i_{0}}}+\sum_{j=1}^{m}\left(p_{i_{0} j}+q_{i_{0} j} k_{i_{0} j}(\lambda)\right) \eta_{j}<-\lambda \xi_{i_{0}}<0$. Therefore, we have

$$
D^{+} u_{i_{0}}\left(t^{*}\right)<-\lambda \xi_{i_{0}}(\kappa+\varepsilon) \mathrm{e}^{-\lambda\left(t^{*}-a\right)}=\dot{z}_{i_{0}}\left(t^{*}\right),
$$

which contradicts the inequality $D^{+} u_{i_{0}}\left(t^{*}\right) \geq \dot{z}_{i_{0}}\left(t^{*}\right)$ in (13). Thus (12) holds for all $t$ $\in[a, b)$. Letting $\varepsilon \rightarrow 0$, we have

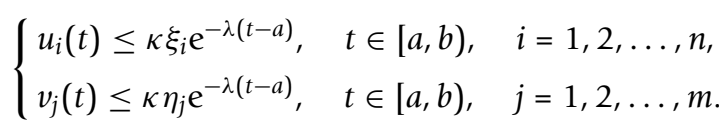

The proof is completed.

Remark 1. Lemma 3 is a generalization of the famous Halanay inequality.

Theorem 2 Under assumptions (H1)-(H3), if the following conditions hold,

(C1) there exist vectors $\xi=\left(\xi_{1}, \xi_{2}, \ldots, \xi_{n}\right)^{T}>0, \eta=\left(\eta_{1}, \eta_{2}, \ldots, \eta_{m}\right)^{T}>0$ and positive number $\lambda>0$ such that

$$
\left\{\begin{array}{l}
\left(\lambda-a_{i} e^{\lambda \delta_{i}}\right) \xi_{i}+\sum_{j=1}^{m}\left[\left|a_{i j}\right|+\left(\left|\alpha_{i j}\right|+\left|\tilde{\alpha}_{i j}\right|\right) k_{i j}(\lambda)\right] G_{j} \eta_{j}<0, i=1,2, \ldots, n, \\
\left(\lambda-b_{j} e^{\lambda \theta_{j}}\right) \eta_{j}+\sum_{i=1}^{n}\left[\left|b_{j i}\right|+\left(\left|\beta_{j i}\right|+\left|\tilde{\beta}_{j i}\right|\right) \bar{k}_{j i}(\lambda)\right] F_{i} \xi_{i}<0, j=1,2, \ldots, m ;
\end{array}\right.
$$

(C2) $\mu=\sup _{k \in N}\left\{\frac{\ln \mu_{k}}{t_{k}-t_{k-1}}\right\}<\lambda$, where $\mu_{k}=\max _{1 \leq i \leq n, 1 \leq j \leq m}\left\{1, \gamma_{i k}, \bar{\gamma}_{j k}\right\}, k \in N$,

then system (1) has exactly one globally exponentially stable equilibrium point, and its exponential convergence rate equals $\lambda-\mu$. 
Proof. Since (C1) holds, from Theorem 1, we know that system (1) has one unique equilibrium point $\left(x_{1}^{*}, \ldots, x_{n}^{*}, y_{1}^{*}, \ldots, y_{m}^{*}\right)^{T}$. Now, we assume that $\left(x_{1}(t), \ldots, x_{n}(t), y_{1}(t), \ldots\right.$, $\left.y_{m}(t)\right)^{T}$ is any solution of system (1), let $\bar{x}_{i}(t)=x_{i}(t)-x_{i}^{*}, \quad i=1,2, \ldots, n$, $\bar{y}_{j}(t)=y_{j}(t)-y_{j}^{*}, j=1,2, \ldots, m$. It is easy to see that system (1) can be transformed into the following system

$$
\begin{aligned}
& \int \dot{\bar{x}}_{i}(t)=-a_{i} \bar{x}_{i}\left(t-\delta_{i}\right)+\sum_{j=1}^{m} a_{i j}\left(g_{j}\left(\bar{y}_{j}(t)+\gamma_{j}^{*}\right)-g_{j}\left(\gamma_{j}^{*}\right)\right) \\
& +\wedge_{j=1}^{m} \alpha_{i j} \int_{0}^{+\infty} K_{i j}(s) g_{j}\left(\bar{y}_{j}(t-s)+\gamma_{j}^{*}\right) \mathrm{d} s-\stackrel{\wedge}{j=1}_{\wedge_{i j}} \alpha_{i j} \int_{0}^{+\infty} K_{j i}(s) g_{j}\left(\gamma_{j}^{*}\right) \mathrm{d} s \\
& +\bigvee_{j=1}^{m} \tilde{\alpha}_{i j} \int_{0}^{+\infty} K_{i j}(s) g_{j}\left(\bar{\gamma}_{j}(t-s)+\gamma_{j}^{*}\right) \mathrm{d} s-\underbrace{m}_{j=1} \tilde{\alpha}_{i j} \int_{0}^{+\infty} K_{i j}(s) g_{j}\left(\gamma_{j}^{*}\right) \mathrm{d} s, \\
& t \neq t_{k}, \\
& \begin{array}{l}
\bar{x}_{i}\left(t_{k}^{+}\right)=\tilde{P}_{i k}\left(\bar{x}_{i}\left(t_{k}^{-}\right)\right), \quad k \in N \\
\dot{\bar{y}}_{j}(t)=-b_{j} \bar{y}_{j}\left(t-\theta_{j}\right)+\sum_{i=1}^{n} b_{j i}\left(f_{i}\left(\bar{x}_{i}(t)+x_{i}^{*}\right)-f_{i}\left(x_{i}^{*}\right)\right)
\end{array} \\
& +\wedge_{i=1}^{n} \beta_{j i} \int_{0}^{+\infty} \bar{K}_{j i}(s) f_{i}\left(\bar{x}_{i}(t-s)+x_{i}^{*}\right) \mathrm{d} s-\bigwedge_{i=1}^{n} \beta_{j i} \int_{0}^{+\infty} \bar{K}_{j i}(s) f_{i}\left(x_{i}^{*}\right) \mathrm{d} s \\
& +\bigvee_{i=1}^{n} \tilde{\beta}_{j i} \int_{0}^{+\infty} \bar{K}_{i j}(s) f_{i}\left(\bar{x}_{i}(t-s)+x_{i}^{*}\right) \mathrm{d} s-\bigvee_{i=1}^{n} \tilde{\beta}_{j i} \int_{0}^{+\infty} \bar{K}_{i j}(s) f_{i}\left(x_{i}^{*}\right) \mathrm{d} s, \\
& t \neq t_{k}, \\
& \bar{\gamma}_{j}\left(t_{k}^{+}\right)=\tilde{Q}_{j k}\left(y_{j}\left(t_{k}^{-}\right)\right), \quad k \in N,
\end{aligned}
$$

where $\tilde{P}_{i k}\left(\bar{x}_{i}(t)\right)=\bar{P}_{i k}\left(\bar{x}_{i}(t)+x_{i}^{*}\right)-\bar{P}_{i k}\left(x_{i}^{*}\right), \quad \tilde{Q}_{j k}\left(\bar{y}_{j}(t)\right)=\bar{Q}_{j k}\left(\bar{y}_{j}(t)+\gamma_{j}^{*}\right)-\bar{Q}_{j k}\left(\gamma_{j}^{*}\right)$, and the initial conditions of (14) are

$$
\begin{cases}\tilde{\phi}(s)=x(s)-x^{*}=\phi(s)-x^{*}, & s \in(-\infty, 0], \\ \tilde{\varphi}(s)=\gamma(s)-y^{*}=\varphi(s)-y^{*}, & s \in(-\infty, 0] .\end{cases}
$$

From (H1) and Lemma 2, we calculate the upper right derivative along the solutions of first equation and third equation of (14), we can obtain

$$
\left\{\begin{aligned}
D^{+}\left|\bar{x}_{i}(t)\right| \leq & -a_{i}\left|\bar{x}_{i}\left(t-\delta_{i}\right)\right|+\sum_{j=1}^{m}\left|a_{i j}\right| G_{j}\left|\bar{y}_{j}(t)\right| \\
& +\sum_{j=1}^{m}\left(\left|\alpha_{i j}\right|+\left|\tilde{\alpha}_{i j}\right|\right) G_{j} \int_{0}^{+\infty}\left|K_{i j}(s)\right|\left|\bar{y}_{j}(t-s)\right| \mathrm{d} s, \\
D^{+}\left|\bar{y}_{j}(t)\right| \leq & -b_{j}\left|\bar{y}_{j}\left(t-\theta_{j}\right)\right|+\sum_{i=1}^{n}\left|b_{j i}\right| F_{i}\left|\bar{x}_{i}(t)\right| \\
& +\sum_{i=1}^{n}\left(\left|\beta_{j i}\right|+\left|\tilde{\beta}_{j i}\right|\right) F_{i} \int_{0}^{+\infty}\left|\bar{K}_{j i}(s)\right|\left|\bar{x}_{i}(t-s)\right| \mathrm{d} s
\end{aligned}\right.
$$


for $i=1,2, \ldots, n, j=1,2, \ldots, m$.

Let $u_{i}(t)=\left|\bar{x}_{i}(t)\right|, \quad v_{j}(t)=\left|\bar{y}_{j}(t)\right|, \quad r_{i}=a_{i}, \quad p_{i j}=\left|a_{i j}\right| G_{j}, \quad q_{i j}=\left(\left|\alpha_{i j}\right|+\left|\tilde{\alpha}_{i j}\right|\right) G_{j}, \quad \bar{r}_{j}=b_{j}$, $\bar{q}_{j i}=\left(\left|\beta_{j i}\right|+\left|\tilde{\beta}_{j i}\right|\right) F_{i}(i=1,2, \ldots, n ; j=1,2, \ldots, m)$, $\bar{q}_{j i}=\left(\left|\beta_{j i}\right|+\left|\tilde{\beta}_{j i}\right|\right) F_{i}(i=1,2, \ldots, n ; j=1,2, \ldots, m)$, then we have

$$
\left\{\begin{array}{l}
D^{+} u_{i}(t) \leq-r_{i} u_{i}\left(t-\delta_{i}\right)+\sum_{j=1}^{m} p_{i j} v_{j}(t)+\sum_{j=1}^{m} q_{i j} \int_{0}^{+\infty}\left|K_{i j}(s)\right| v_{j}(t-s) \mathrm{d} s, \\
D^{+} v_{j}(t) \leq-\bar{r}_{j} v_{j}\left(t-\theta_{j}\right)+\sum_{i=1}^{n} \bar{p}_{j i} u_{i}(t)+\sum_{i=1}^{n} \bar{q}_{j i} \int_{0}^{+\infty}\left|\bar{K}_{j i}(s)\right| u_{i}(t-s) \mathrm{d} s
\end{array}\right.
$$

for $i=1,2, \ldots, n, j=1,2, \ldots, m$, and from $(\mathrm{C} 1)$, there exist vectors $\xi=\left(\xi_{1}, \xi_{2}, \ldots, \xi_{n}\right)^{T}$ $>0, \eta=\left(\eta_{1}, \eta_{2}, \ldots, \eta_{m}\right)^{T}>0$ and positive number $\lambda>0$ such that

$$
\left\{\begin{array}{l}
\left(\lambda-r_{i} \mathrm{e}^{\lambda \delta_{i}}\right) \xi_{i}+\sum_{j=1}^{m}\left[p_{i j}+q_{i j} k_{i j}(\lambda)\right] G_{j} \eta_{j}<0, i=1,2, \ldots, n, \\
\left(\lambda-\bar{r}_{j} \mathrm{e}^{\lambda \theta_{j}}\right) \eta_{j}+\sum_{i=1}^{n}\left[\bar{p}_{j i}+\bar{q}_{j i} \bar{k}_{j i}(\lambda)\right] F_{i} \xi_{i}<0, j=1,2, \ldots, m .
\end{array}\right.
$$

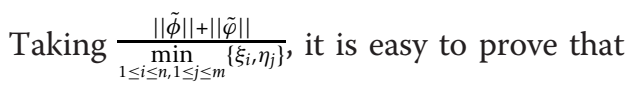

$$
\left\{\begin{array}{l}
u(t) \leq \kappa \xi \mathrm{e}^{-\lambda t},-\infty \leq t \leq 0=t_{0} \\
v(t) \leq \kappa \eta \mathrm{e}^{-\lambda t},-\infty \leq t \leq 0=t_{0}
\end{array}\right.
$$

From Lemma 3, we obtain that

$$
\left\{\begin{array}{l}
u(t) \leq \kappa \xi \mathrm{e}^{-\lambda t}, t_{0} \leq t<t_{1} \\
v(t) \leq \kappa \eta \mathrm{e}^{-\lambda t}, t_{0} \leq t<t_{1} .
\end{array}\right.
$$

Suppose that for $l \leq k$, the inequalities

$$
\left\{\begin{array}{l}
u(t) \leq \kappa \mu_{0} \mu_{1} \ldots \mu_{l-1} \xi \mathrm{e}^{-\lambda t}, t_{l-1} \leq t<t_{l} \\
v(t) \leq \kappa \mu_{0} \mu_{1} \ldots \mu_{l-1} \eta \mathrm{e}^{-\lambda t}, t_{l-1} \leq t<t_{l}
\end{array}\right.
$$

hold, where $\mu_{0}=1$. When $l=k+1$, we note that

$$
\begin{aligned}
u\left(t_{k}\right)=\left|\tilde{P}_{k}\left(u\left(t_{k}^{-}\right)\right)\right| \leq \Gamma_{k} u\left(t_{k}^{-}\right) & \leq \kappa \mu_{0} \mu_{1} \ldots \mu_{k-1} \Gamma_{k} \xi \lim _{t \rightarrow t_{k}^{-}} \mathrm{e}^{-\lambda t} \\
& \leq \kappa \mu_{0} \mu_{1} \ldots \mu_{k-1} \mu_{k} \xi \mathrm{e}^{-\lambda t_{k}}
\end{aligned}
$$

and

$$
\begin{aligned}
v\left(t_{k}\right)=\left|\tilde{Q}_{k}\left(v\left(t_{k}^{-}\right)\right)\right| \leq \bar{\Gamma}_{k} v\left(t_{k}^{-}\right) & \leq \kappa \mu_{0} \mu_{1} \ldots \mu_{k-1} \bar{\Gamma}_{k} \eta \lim _{t \rightarrow t_{k}^{-}} \mathrm{e}^{-\lambda t} \\
& \leq \kappa \mu_{0} \mu_{1} \ldots \mu_{k-1} \mu_{k} \eta \mathrm{e}^{-\lambda t_{k}} .
\end{aligned}
$$

From (20), (21) and $\mu_{k} \geq 1$, we have

$$
\left\{\begin{array}{l}
u(t) \leq \kappa \mu_{0} \mu_{1} \ldots \mu_{k-1} \mu_{k} \xi e^{-\lambda t},-\infty \leq t \leq t_{k \prime} \\
v(t) \leq \kappa \mu_{0} \mu_{1} \ldots \mu_{k-1} \mu_{k} \eta e^{-\lambda t},-\infty \leq t \leq t_{k} .
\end{array}\right.
$$


Combining (15),(16),(22) and Lemma 3, we obtain that

$$
\left\{\begin{array}{l}
u(t) \leq \kappa \mu_{0} \mu_{1} \ldots \mu_{k} \xi e^{-\lambda t}, t_{k} \leq t<t_{k+1} \\
v(t) \leq \kappa \mu_{0} \mu_{1} \ldots \mu_{k} \eta e^{-\lambda t}, t_{k} \leq t<t_{k+1}
\end{array}\right.
$$

Applying the mathematical induction, we can obtain the following inequalities

$$
\left\{\begin{array}{l}
u(t) \leq \kappa \mu_{0} \mu_{1} \ldots \mu_{k} \xi e^{-\lambda t}, t \in\left[t_{k}, t_{k+1}\right), k \in N \\
v(t) \leq \kappa \mu_{0} \mu_{1} \ldots \mu_{k} \eta e^{-\lambda t}, t \in\left[t_{k}, t_{k+1}\right), k \in N
\end{array}\right.
$$

According to (C2), we have $\mu_{k} \leq \mathrm{e}^{\mu\left(t_{k}-t_{k-1}\right)}<\mathrm{e}^{\lambda\left(t_{k}-t_{k-1}\right)}$, so we have

$$
\begin{aligned}
u(t) & \leq \kappa \mathrm{e}^{\mu t_{1}} \mathrm{e}^{\mu\left(t_{2}-t_{1}\right)} \ldots \mathrm{e}^{\mu\left(t_{k-1}-t_{k-2}\right)} \xi \mathrm{e}^{-\lambda t} \\
& =\kappa \xi \mathrm{e}^{\mu t_{k-1}} \mathrm{e}^{-\lambda t} \leq \kappa \xi \mathrm{e}^{-(\lambda-\mu) t}, \quad t \in\left[t_{k-1}, t_{k}\right), \quad k \in N,
\end{aligned}
$$

and

$$
\begin{aligned}
v(t) & \leq \kappa \mathrm{e}^{\mu t_{1}} \mathrm{e}^{\mu\left(t_{2}-t_{1}\right)} \ldots \mathrm{e}^{\mu\left(t_{k-1}-t_{k-2}\right)} \eta \mathrm{e}^{-\lambda t} \\
& =\kappa \eta \mathrm{e}^{\mu t_{k-1}} \mathrm{e}^{-\lambda t} \leq \kappa \eta \mathrm{e}^{-(\lambda-\mu) t}, \quad t \in\left[t_{k-1}, t_{k}\right), \quad k \in N .
\end{aligned}
$$

That is

$$
\left\{\begin{array}{l}
u(t) \leq \kappa \xi e^{-(\lambda-\mu) t}, t \in\left(-\infty, t_{k}\right), k \in N \\
v(t) \leq \kappa \eta e^{-(\lambda-\mu) t}, t \in\left(-\infty, t_{k}\right), k \in N
\end{array}\right.
$$

It follows that

$$
\begin{aligned}
\sum_{i=1}^{n}\left|x_{i}(t)-x_{i}^{*}\right|+\sum_{j=1}^{m}\left|y_{j}(t)-y_{j}^{*}\right| & =\sum_{i=1}^{n} u_{i}(t)+\sum_{j=1}^{m} v_{j}(t) \\
& \leq \sum_{i=1}^{n} \kappa \xi_{i} e^{-(\lambda-\mu) t}+\sum_{j=1}^{m} \kappa \eta_{j} e^{-(\lambda-\mu) t} \\
& =\frac{\sum_{i=1}^{n} \xi_{i}+\sum_{j=1}^{m} \eta_{j}}{\min _{1 \leq i \leq n, 1 \leq j \leq m}\left\{\xi_{i}, \eta_{j}\right\}}(\|\tilde{\phi}\|+\|\tilde{\varphi}\|) \mathrm{e}^{-(\lambda-\mu) t} \\
& =M\left(\left\|\phi-x^{*}\right\|+\left\|\varphi-\gamma^{*}\right\|\right) \mathrm{e}^{-(\lambda-\mu) t},
\end{aligned}
$$

where $M=\frac{\sum_{i=1}^{n} \xi_{i}+\sum_{j=1}^{m} \eta_{j}}{\min _{1 \leq i \leq n, 1 \leq j \leq m}\left\{\xi_{i}, \eta_{j}\right\}}$, then we have

$$
\left\|x(t)-x^{*}\right\|+\left\|\gamma(t)-y^{*}\right\| \leq M\left(\left\|\phi-x^{*}\right\|+\left\|\varphi-\gamma^{*}\right\|\right) e^{-(\lambda-\mu) t} .
$$

The proof is completed.

Remark 2. In Theorem 2, the parameters $\mu_{k}$ and $\mu$ depend on the impulsive disturbance of system (1), and $\lambda$ is actually an estimate of exponential convergence rate of continuous system (2), which depends on the delay kernel functions and system parameters. In order to obtain more precise estimate of the exponential convergence rate of system (1) (or system (2)), we suggest the following optimization problem:

$$
\text { (OP) }\left\{\begin{array}{l}
\max \lambda \\
\text { s.t. }(\mathrm{C} 1) \text { holds. }
\end{array}\right.
$$


Obviously, for continuous system (2), we can immediately obtain the following corollaries.

Corollary 2 Under assumptions (H1) and (H2), if condition (C1) holds, then system (2) has exactly one globally exponentially stable equilibrium point, and its exponential convergence rate equals $\lambda$.

Corollary 3 Under assumptions (H1) and (H2), system (2) has exactly one globally exponentially stable equilibrium point if $C-T L$ is a nonsingular M-matrix.

Remark 3. Note that Lemma 2 transforms the fuzzy AND $(\Lambda)$ and the fuzzy OR $(v)$ operation into the SUM operation $(\Sigma)$. So above results can be applied to the following classical impulsive BAM neural networks with time delays in the leakage terms and distributed delays:

$$
\left\{\begin{aligned}
\dot{x}_{i}(t)= & -a_{i} x_{i}\left(t-\delta_{i}\right)+\sum_{j=1}^{m} a_{i j} g_{j}\left(y_{j}(t)\right) \\
& +\sum_{j=1}^{m} \alpha_{i j} \int_{0}^{+\infty} K_{i j}(s) g_{j}\left(y_{j}(t-s)\right) \mathrm{d} s+I_{i}, \quad t \neq t_{k} \\
x_{i}\left(t^{+}\right)= & x_{i}\left(t^{-}\right)+P_{i k}\left(x_{i}\left(t^{-}\right)\right), \quad t=t_{k}, \quad k \in N, \\
\dot{y}_{j}(t)= & -b_{j} y_{j}\left(t-\theta_{j}\right)+\sum_{i=1}^{n} b_{j i} f_{i}\left(x_{i}(t)\right) \\
& +\sum_{i=1}^{n} \beta_{j i} \int_{0}^{+\infty} \bar{K}_{j i}(s) f_{i}\left(x_{i}(t-s)\right) \mathrm{d} s+J_{j,} \quad t \neq t_{k} \\
y_{j}\left(t^{+}\right)= & y_{j}\left(t^{-}\right)+Q_{j k}\left(y_{j}\left(t^{-}\right)\right), \quad t=t_{k,} \quad k \in N
\end{aligned}\right.
$$

for $i=1,2, \ldots, n ; j=1,2, \ldots, m$.

For model (26), it is easy to obtain the following result:

Theorem 3 Under assumptions (H1)-(H3), if the following conditions hold,

(C1') there exist vectors $\xi=\left(\xi_{1}, \xi_{2}, \ldots, \xi_{n}\right)^{T}>0, \eta=\left(\eta_{1}, \eta_{2}, \ldots, \eta_{m}\right)^{T}>0$ and positive number $\lambda>0$ such that

$$
\left\{\begin{array}{l}
\left(\lambda-a_{i} e^{\lambda \delta_{i}}\right) \xi_{i}+\sum_{j=1}^{m}\left(\left|a_{i j}\right|+\left|\alpha_{i j}\right| k_{i j}(\lambda)\right) G_{j} \eta_{j}<0, \quad i=1,2, \ldots, n, \\
\left(\lambda-b_{j} e^{\lambda \theta_{j}}\right) \eta_{j}+\sum_{i=1}^{n}\left(\left|b_{j i}\right|+\left|\beta_{j i}\right| \bar{k}_{j i}(\lambda)\right) F_{i} \xi_{i}<0, \quad j=1,2, \ldots, m ;
\end{array}\right.
$$

(C2) $\mu=\sup _{k \in N}\left\{\frac{\ln \mu_{k}}{t_{k}-t_{k-1}}\right\}<\lambda$, where $\mu_{k}=\max _{1 \leq i \leq n, 1 \leq j \leq m}\left\{1, \gamma_{i k}, \bar{\gamma}_{j k}\right\}, k \in N$,

then system (26) has exactly one globally exponentially stable equilibrium point, and its exponential convergence rate equals $\lambda-\mu$.

\section{An illustrative example}

In order to illustrate the feasibility of our above-established criteria in the preceding sections, we provide a concrete example. Although the selection of the coefficients and functions in the example is somewhat artificial, the possible application of our theoretical theory is clearly expressed. 
Example. Consider the following impulsive BAM FCNNs with time delays in the leakage terms and distributed delays:

$$
\begin{aligned}
& \int \dot{x}_{i}(t)=-a_{i} x_{i}\left(t-\delta_{i}\right)+\sum_{j=1}^{2} a_{i j} g_{j}\left(y_{j}(t)\right)+\sum_{j=1}^{2} \tilde{a}_{i j} v_{j}+I_{i} \\
& +\underset{j=1}{2} \alpha_{i j} \int_{0}^{+\infty} K_{i j}(s) g_{j}\left(y_{j}(t-s)\right) \mathrm{d} s+\underset{j=1}{2} \tilde{\alpha}_{i j} \int_{0}^{+\infty} K_{i j}(s) g_{j}\left(y_{j}(t-s)\right) \mathrm{d} s \\
& +\stackrel{\wedge}{j=1}^{2} T_{i j} v_{j}+\underset{j=1}{\vee 2} H_{i j} v_{j,} \quad t \neq t_{k} \\
& \left\{\begin{array}{l}
x_{i}\left(t^{+}\right)=x_{i}\left(t^{-}\right)+P_{i k}\left(x_{i}\left(t^{-}\right)\right)=x_{i}\left(t^{-}\right)-\left(1+\mathrm{e}^{0.125 k}\right)\left(x_{i}\left(t^{-}\right)-1\right), t=t_{k \prime} \\
\dot{y}_{j}(t)=-b_{j} y_{j}\left(t-\theta_{j}\right)+\sum_{i=1}^{2} b_{j i} f_{i}\left(x_{i}(t)\right)+\sum_{i=1}^{2} \tilde{b}_{j i} u_{i}+J_{j}
\end{array}\right. \\
& +\underset{i=1}{2} \beta_{j i} \int_{0}^{+\infty} \bar{K}_{j i}(s) f_{i}\left(x_{i}(t-s)\right) \mathrm{d} s+\underset{i=1}{\vee} \tilde{\beta}_{j i} \int_{0}^{+\infty} \bar{K}_{i j}(s) f_{i}\left(x_{i}(t-s)\right) \mathrm{d} s \\
& +{ }_{i=1}^{2} \bar{T}_{j i} u_{i}+\underset{i=1}{2} \bar{H}_{j i} u_{i}, t \neq t_{k} \\
& \gamma_{j}\left(t^{+}\right)=\gamma_{j}\left(t^{-}\right)+Q_{j k}\left(\gamma_{j}\left(t^{-}\right)\right)=\gamma_{j}\left(t^{-}\right)-\left(1+\mathrm{e}^{0.125 k}\right)\left(\gamma_{j}\left(t^{-}\right)-1\right), \quad t=t_{k}
\end{aligned}
$$

for $k \in N, i=1,2, j=1,2, t>0, t_{0}=0, t_{k}=t_{k-1}+0.5 k, k \in N$, where

$$
\begin{aligned}
& a_{1}=4.5, \quad a_{2}=4.5, \quad \delta_{1}=0.2, \quad \delta_{2}=0.3, \quad a_{11}=\frac{4}{3}, \quad a_{12}=-\frac{1}{2}, \\
& a_{21}=\frac{1}{2}, \quad a_{22}=\frac{2}{3}, \quad \tilde{a}_{11}=1, \quad \tilde{a}_{12}=-2, \quad \tilde{a}_{21}=-2, \quad \tilde{a}_{22}=1, \\
& I_{1}=\frac{67}{12}, \quad I_{2}=\frac{5}{12}, \quad \alpha_{11}=\frac{1}{3}, \quad \alpha_{12}=-\frac{1}{4}, \quad \alpha_{21}=\frac{1}{4}, \quad \alpha_{22}=\frac{2}{3} \text {, } \\
& \tilde{\alpha}_{11}=\frac{1}{3}, \quad \tilde{\alpha}_{12}=\frac{1}{4}, \quad \tilde{\alpha}_{21}=-\frac{1}{4}, \quad \tilde{\alpha}_{22}=\frac{2}{3}, \quad T_{11}=1, \quad T_{12}=0, \\
& T_{21}=0, \quad T_{22}=1, \quad H_{11}=1, \quad H_{12}=0, \quad H_{21}=0, \quad H_{22}=1 \text {, } \\
& v_{1}=1, \quad v_{2}=2 \\
& b_{1}=4.5, \quad b_{2}=4.5, \quad \theta_{1}=0.2, \quad \theta_{2}=0.1, \quad b_{11}=\frac{1}{3}, \quad b_{12}=-\frac{2}{3}, \\
& b_{21}=\frac{4}{3}, \quad b_{22}=\frac{1}{3}, \quad \tilde{b}_{11}=1, \quad \tilde{b}_{12}=3, \quad \tilde{b}_{21}=2, \quad \tilde{b}_{22}=-2 \text {, } \\
& J_{1}=-\frac{1}{2}, \quad J_{2}=\frac{7}{6}, \quad \beta_{11}=\frac{1}{3}, \quad \beta_{12}=-\frac{1}{6}, \quad \beta_{21}=\frac{1}{3}, \quad \beta_{22}=\frac{1}{3}, \\
& \tilde{\beta}_{11}=\frac{1}{3}, \quad \tilde{\beta}_{12}=\frac{1}{6}, \quad \tilde{\beta}_{21}=\frac{1}{3} \quad \tilde{\beta}_{22}=\frac{1}{3}, \quad \tilde{T}_{11}=1, \quad \tilde{T}_{12}=0, \\
& \tilde{T}_{21}=0, \quad \tilde{T}_{22}=1, \quad \tilde{H}_{11}=1, \quad \tilde{H}_{12}=0, \quad \tilde{H}_{21}=0, \quad \tilde{H}_{22}=1 \text {, } \\
& u_{1}=1, \quad u_{2}=1 \text {; } \\
& K_{i j}(s)=\bar{K}_{i j}(s)=\mathrm{e}^{-s}, \quad f_{i}(s)=g_{j}(s)=\frac{|s+1|-|s-1|}{2}, \quad i, j=1,2 .
\end{aligned}
$$

From above parameters, we have $F_{1}=F_{2}=1, G_{1}=G_{2}=1$, and $\left(k_{i j}(\lambda)\right)_{2 \times 2}=\left(\bar{k}_{j i}(\lambda)\right)_{2 \times 2}=\left(\begin{array}{l}\frac{1}{1-\lambda} \frac{1}{1-\lambda} \\ \frac{1}{1-\lambda} \frac{1}{1-\lambda}\end{array}\right), \quad \Gamma_{k}=\bar{\Gamma}_{k}=\left(\begin{array}{ll}e^{0.125 k} \\ e^{0.125 k}\end{array}\right)$.

Solving the following optimization problem

$$
\left\{\begin{aligned}
\max \lambda & \\
0> & \left(\lambda-a_{1} \mathrm{e}^{\lambda \delta_{1}}\right) \xi_{1}+\left(\left|a_{11}\right|+\left(\left|\alpha_{11}\right|+\left|\tilde{\alpha}_{11}\right|\right) k_{11}(\lambda)\right) G_{1} \eta_{1} \\
& +\left(\left|a_{12}\right|+\left(\left|\alpha_{12}\right|+\left|\tilde{\alpha}_{12}\right|\right) k_{12}(\lambda)\right) G_{2} \eta_{2} \\
0> & \left(\lambda-a_{2} \mathrm{e}^{\lambda \delta_{2}}\right) \xi_{1}+\left(\left|a_{21}\right|+\left(\left|\alpha_{21}\right|+\left|\tilde{\alpha}_{21}\right|\right) k_{21}(\lambda)\right) G_{1} \eta_{1} \\
& +\left(\left|a_{22}\right|+\left(\left|\alpha_{22}\right|+\left|\tilde{\alpha}_{22}\right|\right) k_{22}(\lambda)\right) G_{2} \eta_{2} \\
0> & \left(\lambda-b_{1} \mathrm{e}^{\lambda \theta_{1}}\right) \eta_{1}+\left(\left|b_{11}\right|+\left(\left|\beta_{11}\right|+\left|\tilde{\beta}_{11}\right|\right) \bar{k}_{11}(\lambda)\right) F_{1} \xi_{1} \\
& +\left(\left|b_{12}\right|+\left(\left|\beta_{12}\right|+\left|\tilde{\beta}_{12}\right|\right) \bar{k}_{12}(\lambda)\right) F_{2} \xi_{2} \\
0> & \left(\lambda-b_{2} \mathrm{e}^{\lambda \theta_{2}}\right) \eta_{2}+\left(\left|b_{21}\right|+\left(\left|\beta_{21}\right|+\left|\tilde{\beta}_{21}\right|\right) \bar{k}_{21}(\lambda)\right) F_{1} \xi_{1} \\
& +\left(\left|b_{22}\right|+\left(\left|\beta_{22}\right|+\left|\tilde{\beta}_{22}\right|\right) \bar{k}_{22}(\lambda)\right) F_{2} \xi_{2 \prime} \\
\lambda> & 0, \quad \xi=\left(\xi_{1}, \xi_{2}\right)^{T}>0, \quad \eta=\left(\eta_{1}, \eta_{2}\right)^{T}>0 .
\end{aligned}\right.
$$


We obtain that $\lambda \approx 0.3868>0, \xi=(1082041,1327618)^{T}>0$ and $\eta=(716212$, $1050021)^{T}>0$, so $(\mathrm{C} 1)$ holds. From Theorem 1 , we know system $(27)$ has a unique equilibrium point, this equilibrium point is $(1,1,1,1)^{T}$. Also,

$$
\begin{aligned}
\mu_{k} & =\max _{1 \leq i \leq 2,1 \leq j \leq 2}\left\{1, \gamma_{i k}, \bar{\gamma}_{j k}\right\}=e^{0.125 k}, \\
\mu & =\sup _{k \in N} \frac{\ln \mu_{k}}{t_{k}-t_{k-1}}=\frac{0.125 k}{0.5 k}=0.25<0.3868=\lambda .
\end{aligned}
$$

That is, (C2) holds. From Theorem 2, the unique equilibrium point $(1,1,1,1)^{T}$ of system (27) is globally exponentially stable, and its exponential convergence rate is about 0.1368. The numerical simulation is shown in Figure 1 and 2.

\section{Conclusions}

In this paper, a class of impulsive BAM FCNNs with time delays in the leakage terms and distributed delays has been formulated and investigated. Some new criteria on the existence, uniqueness and global exponential stability of equilibrium point for the networks have been derived by using $M$-matrix theory and the impulsive delay integro-differential inequality. Our stability criteria are delay-dependent and impulse-dependent. The neuronal output activation functions and the impulsive operators only need to are Lipschitz continuous, but need not to be bounded and monotonically increasing. Some restrictions of delay kernel functions are also removed. It is worthwhile to mention that our technical methods are practical, in the sense that all new stability conditions are stated in simple algebraic forms and provided a more precise estimate of the exponential convergence rate, so their verification and applications are straightforward and

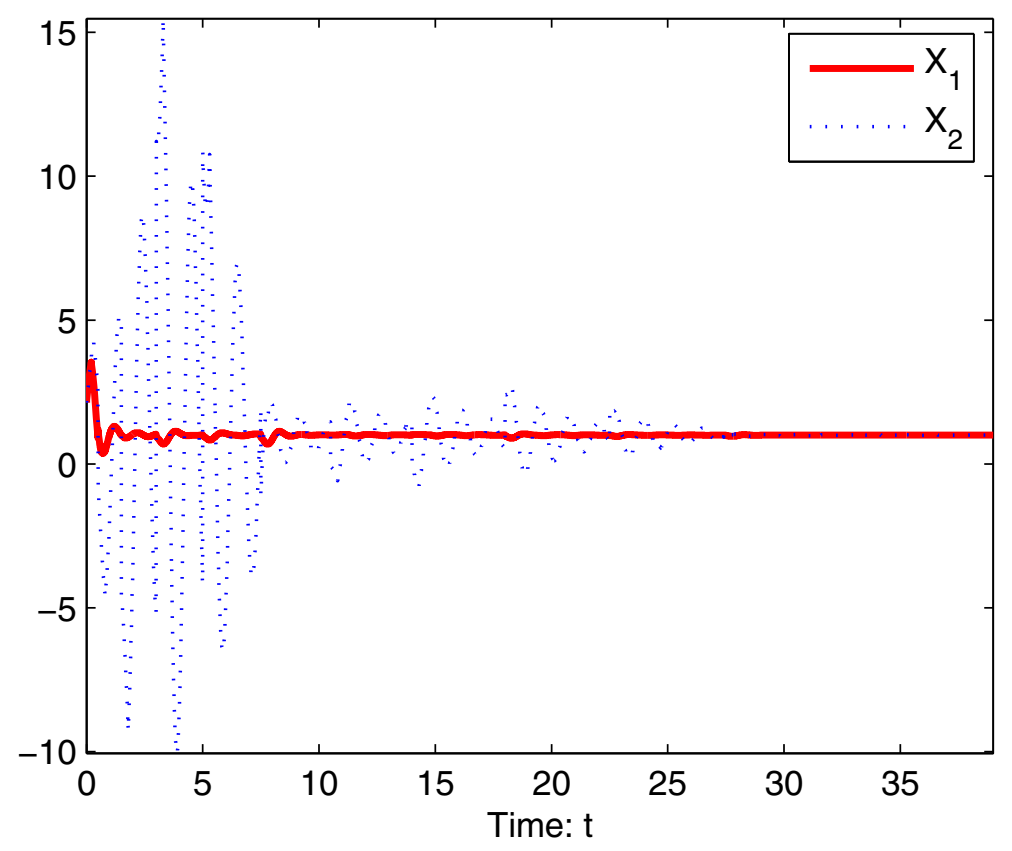

Figure 1 Behavior of the state variable $x(t)$ with time impulses 


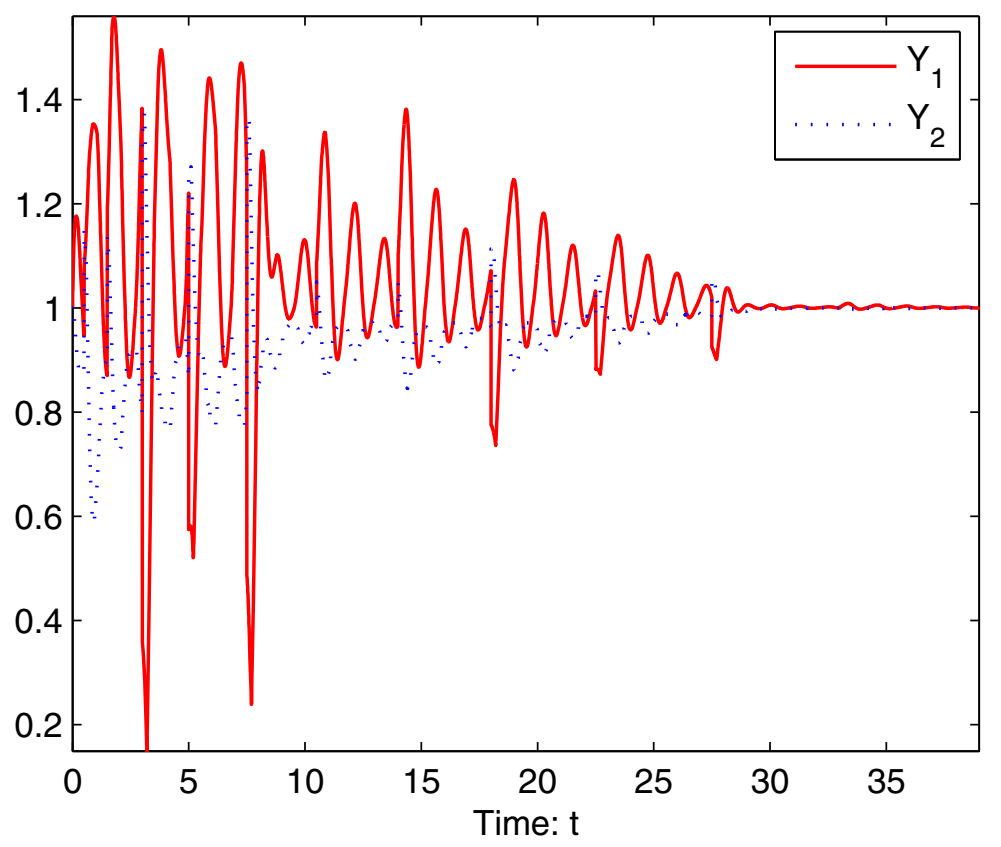

Figure 2 Behavior of the state variable $y(t)$ with time impulses

convenient. The effectiveness of our results has been demonstrated by the convenient numerical example.

\section{Acknowledgements}

This work is supported by the Scientific Research Fund of Sichuan Provincial Education Department under Grant $092 C 057$.

\section{Authors' contributions}

ZX designed and performed all the steps of proof in this research and also wrote the paper. $\mathrm{KL}$ participated in the design of the study and helped to draft and revise manuscript. All authors read and approved the final manuscript.

\section{Competing interests}

The authors declare that they have no competing interests.

\section{Received: 25 May 2011 Accepted: 1 September 2011 Published: 1 September 2011}

\section{References}

1. Kosko, B: Bi-directional associative memories. IEEE Trans Syst Man Cybern. 18, $49-60$ (1988). doi:10.1109/21.87054

2. Mathai, G, Upadhyaya, BR: Performance analysis and application of the bidirectional associative memory to industrial spectral signatures. Proceedings of the IJCNN-1989. 1, 33-37 (1989)

3. Li, C, Liao, X, Zhang, R: Delay-dependent exponential stability analysis of bi-directional associative memory neural networks with time delay: an LMI approach. Chaos Solitons Fractals. 24, 1119-1134 (2005). doi:10.1016/j. chaos.2004.09.052

4. Arik, S: Global asymptotic stability analysis of bidirectional associative memory neural networks with time delays. IEEE Trans Neural Netw. 16, 580-586 (2005). doi:10.1109/TNN.2005.844910

5. Gopalsamy, K, He, X: Delay-independent stability in bi-directional associative memory with axonal signal transmission delays. IEEE Trans Neural Netw. 5, 998-1002 (1994). doi:10.1109/72.329700

6. Mohamad, S: Global exponential stability in continuous-time and discrete-time delay bidirectional neural networks. Phys D. 159, 233-251 (2001). doi:10.1016/S0167-2789(01)00344-X

7. Zhang, J, Yang, Y: Global stability analysis of bi-directional associative memory neural networks with time delay. Int J Circ Theory Appl. 29, 185-196 (2001). doi:10.1002/cta.144

8. Liang, J, Cao, J: Exponential stability of continuous-time and discrete-time bidirectional associative memory networks with delays. Chaos Solitons Fractals. 22, 773-785 (2004). doi:10.1016/j.chaos.2004.03.004

9. Feng, C, Plamondon, R: Stability analysis of bidirectional associative memory networks with time delays. IEEE Trans Neural Netw. 14, 1560-1565 (2003). doi:10.1109/TNN.2003.820829 
10. Rao, VSH, Phaneendra, BhRM: Global dynamics of bidirectional associative memory neural networks involving transmission delays and dead zones. Neural Netw. 12, 455-465 (1999). doi:10.1016/50893-6080(98)00134-8

11. Song, Q, Cao, J: Global exponential stability of bidirectional associative memory neural networks with distributed delays. J Comput Appl Math. 202, 266-279 (2007). doi:10.1016/j.cam.2006.02.031

12. Gopalsamy, K: Stability and Oscillations in Delay Differential Equations of Population Dynamics. Kluwer, Dordrecht (1992)

13. Gopalsamy, K: Leakage delays in BAM. J Math Anal Appl. 325, 1117-1132 (2007). doi:10.1016/j.jmaa.2006.02.039

14. Peng, S: Global attractive periodic solutions of BAM neural networks with continuously distributed delays in the leakage terms. Nonlinear Anal Real World Appl. 11, 2141-2151 (2010). doi:10.1016/j.nonrwa.2009.06.004

15. Li, X, Fu, X, Balasubramaniam, P, Rakkiyappan, R: Existence, uniqueness and stability analysis of recurrent neural networks with time delay in the leakage term under impulsive perturbations. Nonlinear Anal Real World Appl. 11, 4092-4108 (2010). doi:10.1016/j.nonrwa.2010.03.014

16. Li, X, Cao, J: Delay-dependent stability of neural networks of neutral type with time delay in the leakage term. Nonlinearity. 23, 1709-1726 (2010). doi:10.1088/0951-7715/23/7/010

17. Balasubramaniam, P, Kalpana, M, Rakkiyappan, R: Global asymptotic stability of BAM fuzzy cellular neural networks with time delay in the leakage term, discrete and unbounded distributed delays. Math Comput Model. 53, 839-853 (2011). doi:10.1016/j.mcm.2010.10.021

18. Li, X, Rakkiyappan, R, Balasubramaniam, P: Existence and global stability analysis of equilibrium of fuzzy cellular neural networks with time delay in the leakage term under impulsive perturbations. J Franklin Institute. 348, 135-155 (2011). doi:10.1016/j.jfranklin.2010.10.009

19. Yang, T, Yang, L, Wu, C, Chua, L: Fuzzy cellular neural networks: theory. Proceedings of IEEE International Workshop on Cellular Neural Networks and Applications. 181-186 (1996)

20. Yang, T, Yang, L: The global stability of fuzzy cellular neural networks. IEEE Trans Cric Syst I. 43, 880-883 (1996). doi:10.1109/81.538999

21. Liu, Y, Tang, W: Exponential stability of fuzzy cellular neural networks with constant and time-varying delays. Phys Lett A. 323, 224-233 (2004). doi:10.1016/j.physleta.2004.01.064

22. Yuan, $\mathrm{K}, \mathrm{Cao}, \mathrm{J}$, Deng, J: Exponential stability and periodic solutions of fuzzy cellular neural networks with time-varying delays. Neurocomputing. 69, 1619-1627 (2006). doi:10.1016/j.neucom.2005.05.011

23. Huang, T: Exponential stability of fuzzy cellular neural networks with distributed delay. Phys Lett A. 351, 48-52 (2006). doi:10.1016/j.physleta.2005.10.060

24. Li, K: Impulsive effect on global exponential stability of BAM fuzzy cellular neural networks with time-varying delays. Int J Syst Sci. 41, 131-142 (2010). doi:10.1080/00207720903042921

25. Song, Q, Cao, J: Dynamical behaviors of discrete-time fuzzy cellular neural networks with variable delays and impulses. J Franklin Institute. 345, 39-59 (2007)

26. Wang, S, Chung, KFL, Fu, D: Applying the improved fuzzy cellular neural network IFCNN to white blood cell detection. Neurocomputing. 70, 1348-1359 (2007). doi:10.1016/j.neucom.2006.07.012

27. Li, K, Zhang, L: Global exponential stability of BAM fuzzy cellular neural networks with distributed delays and impulses. J Appl Math Inform. 29, 211-225 (2011)

28. Akca, H, Alassar, R, Covachev, V, Covacheva, Z, Al-Zahrani, E: Continuous-time additive Hopfield-type neural networks with impulses. J Math Anal Appl. 290, 436-451 (2004). doi:10.1016/j.jmaa.2003.10.005

29. Xu, D, Yang, Z: Impulsive delay differential inequality and stability of neural networks. J Math Anal Appl. 305, 107-120 (2005). doi:10.1016/j.jmaa.2004.10.040

30. Yang, Z, Xu, D: Impulsive effects on stability of Cohen-Grossberg neural networks with variable delays. Appl Math Comput. 177, 63-78 (2006). doi:10.1016/j.amc.2005.10.032

31. Gopalsamy, K: Stability of artificial neural networks with impulses. Appl Math Comput. 154, 783-813 (2004). doi:10.1016/ S0096-3003(03)00750-1

32. Mohamad, S, Gopalsamy, K, Akca, H: Exponential stability of artificial neural networks with distributed delays and large impulses. Nonlinear Anal Real World Appl. 9, 872-888 (2008). doi:10.1016/j.nonrwa.2007.01.011

33. Li, Y: Global exponential stability of BAM neural networks with delays and impulses. Chaos, Solitions Fractals. 24, 279-285 (2005)

34. Li, Y, Yang, C: Global exponential stability analysis on impulsive BAM neural networks with distributed delays. J Math Anal Appl. 324, 1125-1139 (2006). doi:10.1016/j.jmaa.2006.01.016

35. Liu, B, Huang, L: Global exponential stability of BAM neural networks with recent-history distributed delays and impulses. Neurocomputing. 69, 2090-2096 (2006). doi:10.1016/j.neucom.2005.09.014

36. Huang, Z, Luo, X, Yang, Q: Global asymptotic stability analysis of bidirectional associative memory neural networks with distributed delays and impulse. Chaos, Solitons Fractals. 34, 878-885 (2007). doi:10.1016/j.chaos.2006.03.112

37. Zhou, Q: Global exponential stability of BAM neural networks with distributed delays and impulses. Nonlinear Anal Real World Appl. 10, 144-153 (2009). doi:10.1016/..nonrwa.2007.08.019

38. Horn, RA, Johnson, CR: Topics in Matrix Analysis. Cambridge University Press, Cambridge (1991)

39. Cronin, J: Fixed Points and Topological Degree in Nonlinear Analysis. American Mathematical Society, Providence. (1964)

doi:10.1186/1029-242X-2011-43

Cite this article as: Zhang and Li: Integro-differential inequality and stability of BAM FCNNs with time delays in the leakage terms and distributed delays. Journal of Inequalities and Applications 2011 2011:43. 